\title{
RP EBUS as a guide for transbronchial pulmonary biopsy in the diagnosis of organizing pneumonia
}

\author{
Gaetano Cicchitto, ${ }^{1}$ Mario Polverino, ${ }^{1}$ Antonio Capuozzo, ${ }^{1}$ Luigi Cacace, ${ }^{2}$ Antonietta Cavallera, ${ }^{3}$ Imma Mauro, \\ Francesco Ferrigno, ${ }^{1}$ Francesca Polverino ${ }^{4}$ \\ ${ }^{1}$ Department of Pneumology and Endoscopic Unit, Ospedale “M. Scarlato”, Scafati (SA), Italy \\ ${ }^{2}$ Division of Pathology, Ospedale “A. Tortora”, Pagani (SA), Italy \\ ${ }^{3}$ Department of Radiology, Ospedale “M. Scarlato”, Scafati (SA), Italy \\ ${ }^{4}$ Asthma and Airway Disease Center, University of Arizona, Tucson, AZ, USA
}

\begin{abstract}
Diffuse parenchymal lung diseases (DPLDs) include a wide variety of manifestations characterized by different degrees of inflammation and fibrosis with various patterns of secondary lobule alterations, such that the diagnosis often requires histopathological confirmation in addition to clinical and radiological data. Radial probe endobronchial ultrasonography (RP EBUS) can be used as a guide for transbronchial pulmonary biopsy (TBPB) to obtain tissue samples, and thus can be a useful tool in the diagnostic management of peripheral pulmonary lesions. Organizing pneumonia (OP) is a particular type of DPLD characterized by lung inflammation and scarring that obstruct the small airways and air sacs of the lung. In this study, we describe how and when RP EBUS can be used to guide TBPB and significantly help in the diagnosis of OP.
\end{abstract}

Key words: Flexible bronchoscopy; organizing pneumonia; radial probe endobronchial ultrasonography; transbronchial pulmonary biopsy.

Correspondence: Francesca Polverino, Asthma and Airway Disease Research Center, University of Arizona, Tucson, AZ 85717, USA. Tel. +1.520.626-8485.

E-mail: fpolverino@copdnet.org; francy@email.arizona.edu

Contributions: All the authors conducted the study and/or contributed to data analysis and interpretation. All authors contributed to the writing and editing of the manuscript. All the authors have read and approved the final version of the manuscript and agreed to be accountable for all aspects of the work.

Conflict of interest: None of the authors have conflicts of interest to disclose. MP is Coordinator of the Editorial Board of Multidisciplinary Respiratory Medicine; FP is a member of the Editorial Board of Multidisciplinary Respiratory Medicine.

Funding: This work has been supported by funds from the Asthma and Airway Disease Research Center (University of Arizona); Flight Attendants Medical Research Institute grant \#YFAC141004, the Parker B. Francis Foundation Fellowship.

Availability of data and materials: The data used to support the findings of this study are available from the corresponding author on reasonable request.

Ethics approval and consent to participate: The research was conducted in accordance with the World Medical Association Declaration of Helsinki. For all the clinical cases presented in this series, written informed consent from the patients was obtained.

Patient consent for publication: Not applicable.

Informed consent: For all the clinical cases presented in this series, written informed consent from the patients was obtained. 


\section{Introduction}

Diffuse parenchymal lung diseases (DPLDs), also described as interstitial lung diseases (ILDs), encompass a variety of diseases, many of which are uncommon and of unknown etiology $[1,2]$. Because DPLDs show different degrees of inflammation and fibrosis, radiological features may be quite heterogeneous, showing diverse patterns of secondary lobule alterations. Radial probe endobronchial ultrasonography (RP EBUS), a methodology that uses ultrasound properties in order to identify abnormalities in the airways and lung parenchyma, is routinely employed to identify and sample pulmonary nodules and peripheral lung lesions [3]. Recently, RP EBUS was also proposed as a diagnostic tool, in the context of those DPLDs that are radiologically characterized by focal areas of consolidation and/or "ground glass" [4]. Organizing pneumonia (OP) is a reaction pattern characterized radiographically by variable features, and histologically by loose plugs of granulation tissue composed of fibroblasts, myofibroblasts, collagen, and fibrinous exudates included in a myxoid matrix within the distal airspaces (respiratory bronchioles, alveolar ducts and alveoli); the OP is considered to be secondary if the above features are found in a specific clinical context (e.g. neoplasm, pulmonary infection, drug reaction, connective tissue diseases, etc.); otherwise, OP is named cryptogenic [5], being the latter part of the idiopathic interstitial pneumonia (IIP). Differential diagnosis of OP requires histological confirmation because of the non-specificity of clinical and radiological signs associated with it. Surgical biopsy is recommended to obtain a significant tissue sampling in DPLDs $[2,6]$; however, the risks associated with an invasive procedure [7] make safer and more reliable methods desirable. Hereby, we describe three OP cases in which RP EBUS was successfully used as a guide for transbronchial pulmonary biopsy (TBPB).

The research was conducted in accordance with the World Medical Association Declaration of Helsinki. For all the clinical cases presented in this series, written informed consent from the patients was obtained.

\section{Case \#1}

A 54-year-old man was admitted to the hospital for progressive dyspnea, fatigue, non-productive cough, and intermittent fever for about three months; a chest radiograph showed left basal opacities. A previous course of empirical antibiotic therapy had no beneficial effects. The patient had never smoked, showed no symptoms nor signs of connective tissue disease, and reported no occupational exposures. He was under treatment with valsartan $80 \mathrm{mg} /$ die for systemic arterial hypertension, alfuzosin $10 \mathrm{mg} / \mathrm{die}$ for prostatic hypertrophy, and insulin for diabetes mellitus. Physical examination revealed inspiratory crackles on the left basal side. Blood cell count showed a mild leukocytosis $\left(12,000 / \mathrm{mm}^{3}\right)$ and a mild Creactive protein (CRP) increase $(6.8 \mathrm{mg} / \mathrm{l})$; antinuclear antibodies, rheumatoid factor, anti-cyclic citrullinated peptide, anti-double strand DNA, and anti-nucleoprotein antibodies were negative. Pulmonary function tests (PFTs) were normal (TLC: $81 \%$ predicted; FVC: $88 \%$ predicted; $\mathrm{FEV}_{1}: 83 \%$ predicted; $\mathrm{DL}_{\mathrm{CO}}: 88.5 \%$ predicted). A chest Computed Tomography (CT)-scan revealed left alveolar opacities with both consolidative and ground glass (GGOs) features, mainly located in the subpleural areas of the lingula and in the left lung basal region, with peribronchiolar distribution (Figure 1A). An endoscopic procedure was performed under conscious sedation. A flexible videobronchoscope with an external diameter of $6.2 \mathrm{~mm}$ and a working channel of $3.2 \mathrm{~mm}$ (Olympus BF XT 160) was introduced, via oral route, into the patient's airways for bronchial tree inspection and bronchoalveolar lavage (BAL); subsequently, a 20 MHz RP EBUS system (1.7 mm external diameter) was inserted into the working channel of the bronchoscope and directed to the target lung region identified using the chest CT images. A bronchiolar segment leading the probe into the lesion was identified, and transbronchial biopsies were performed, obtaining a total of five lung tissue samples. A mixed "blizzard sign" was observed in the basal left lung (Figure 1B). As described by Izumo et al. [8] a "blizzard sign" is, in general terms, defined as a hyperechoic shadow that is subtly more intense than a typical snowstorm appearance when scanning normal alveolar tissue, whereas a mixed "blizzard sign" is a typical feature of a GGO lesion developing a solid component $[8,9]$. BAL differential cell count showed a mixed pattern alveolitis (macrophages 50\%; lymphocytes $37 \%$; neutrophils $12 \%$; eosinophils $1 \%$ ); bacterial cultures were negative. The transbronchial biopsies displayed mild chronic interstitial inflammation and fibroblastic buds filling distal airways (Figure 1C). OP was diagnosed, and a steroid therapy was initiated.

\section{Case \#2}

A 28-year-old man was admitted to the hospital with a history of subacute flu-like symptoms, dry cough, and dyspnoea for the past two months, not reverted after empirical antibiotic therapy. The patient was a former smoker but did not report any other environmental or occupational exposure. Clinical evaluation and blood tests were normal, apart from unspecific mild leukocytosis $\left(11,500 / \mathrm{mm}^{3}\right)$ and a modest increase of C Reactive Protein (CRP: $9 \mathrm{mg} / \mathrm{l})$. Crackles on the right side were noticed upon auscultation. PFTs showed a mild restrictive ventilatory defect (TLC: $74 \%$ predicted; FVC: $87 \%$ predicted; $\mathrm{FEV}_{1}: 87 \%$ predicted; $\mathrm{DL}_{\mathrm{CO}}: 82.1 \%$ predicted). A chest CT-scan revealed right side alveolar opacities (Figure 2A), some of them surrounded by areas of GGOs. A bronchoscopy was performed as described in Case \#1, and six lung tissue samples were obtained. The lesion was identified as a hypoe-
A

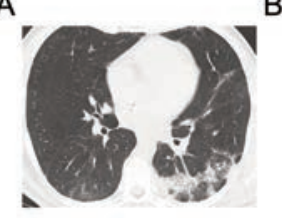

B

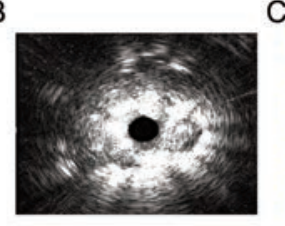

C

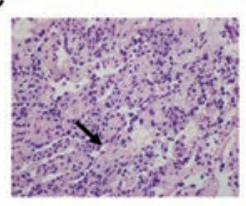

Figure 1. A) Left lung consolidative and ground glass opacities. B) Mixed blizzard sign in the left lower lobe. C) fibroblast embedded in a myxoid matrix filling distal airspaces (hematoxylin and eosin stain, magnification $20 \mathrm{x}$ ).
A

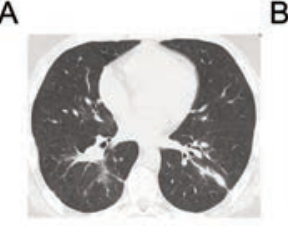

B

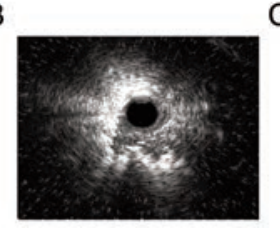

C

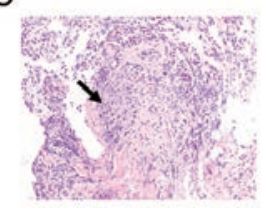

Figure 2. A) Right lower lobe alveolar opacity. B) Hypoechoic lesion in the right lower lobe. C) Loose plugs of granulation tissue filling distal airspaces (hematoxylin and eosin stain, magnification $40 \mathrm{x}$ ). 
choic morphology in the right lower lobe (Figure 2B). BAL differential cell count showed mixed pattern alveolitis (macrophages $50 \%$; lymphocytes $40 \%$; neutrophils $10 \%$ ); bacterial cultures were negative. The transbronchial biopsies displayed mild chronic interstitial inflammation and fibroblastic buds filling distal airways (Figure 2C). OP was diagnosed, and steroid therapy was initiated.

\section{Case \#3}

A 31-year-old non-smoking, North African Caucasian woman suffered from intermittent fever and mild productive cough for about two months prior to the visit. The symptoms did not regress after empirical antibiotic therapy and occasional low-dose steroid treatment. The patient was otherwise healthy with no comorbidities, nor a history of environmental and occupational exposures. Patchy right crackles were noticed upon thoracic auscultation. Blood tests revealed a moderate leukocytosis $\left(15.800 / \mathrm{mm}^{3}\right)$ and an increase in CRP $(17,3 \mathrm{mg} / \mathrm{l})$. PFTs revealed a mild restrictive ventilatory alteration (TLC: $70 \%$ predicted; FVC: $81 \%$ predicted; $\mathrm{FEV}_{1}: 84 \%$ predicted; $\mathrm{DL}_{\mathrm{CO}}: 75 \%$ predicted). Chest $\mathrm{CT}$ showed areas of consolidation in the upper dorsal lobes, bilaterally, with a predominant peribronchiolar and subpleural distribution (Figure 3A). A flexible bronchoscopic procedure was performed as described in Case \#1. A lesion with hypoechoic morphology with hyperechoic dots was identified (Figure 3B). BAL differential cell count showed a mixed pattern alveolitis (macrophages 35\%; lymphocytes 55\%; neutrophils 10\%); bacterial cultures were negative. Via transbronchial biopsies, five lung tissue samples were obtained. The samples displayed an OP pattern characterized by granulation tissue plugs in respiratory bronchioles and alveolar ducts with accompanying chronic interstitial inflammation (Figure 3C). Steroid therapy was therefore initiated.

\section{Discussion and Conclusion}

In this study, we highlight the advantages of RP EBUS TBPB in the differential diagnosis of certain kinds of DPLDs, such as OP.

RP EBUS is widely employed in the diagnostic workup of peripheral pulmonary lesions [3]. The diagnostic yield of RP EBUS-assisted sampling is around $70 \%$, without relevant complications; higher yields are obtained for lesions that are $>2 \mathrm{~cm}$ in size, malignant, and/or associated with a bronchus sign on CT[10]. Since specimens obtained by TBPB originate from the centrilobular region of the secondary pulmonary lobule (i.e. a region where respiratory and terminal bronchioles, as well as lymphatics, are located), pathologies developing in these locations are more easily
A

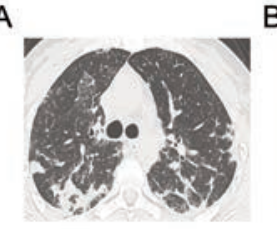

B

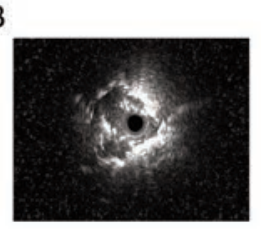

C

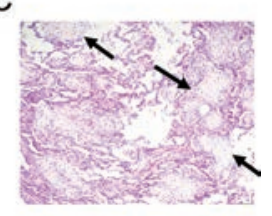

Figure 3. A) Consolidation in the dorsal upper lobes. B) Heterogeneous echogenic lesion in the right upper lobe. C) Granulation tissue plugs in respiratory bronchioles and alveolar ducts with accompanying chronic interstitial inflammation (hematoxylin and eosin stain, magnification $10 \mathrm{x}$ ). diagnosed [11]. To date, only a few studies evaluated the value of RP EBUS-guided TBPB in the differential diagnosis of DPLDs, mostly because lung biopsy is considered the best way to obtain an appropriate tissue specimen [2,6]. The few available studies assessing the role of RP EBUS, performed both by forceps and cryoprobes, as a guide for TBPB in the differential diagnosis of DPLDs [4,12], show that the diagnostic yield of RP EBUS is not always better than the one achieved via lung biopsy, but the risk of complications such as pneumothorax is statistically lower than lung biopsy.

Here, we assessed whether the RP EBUS TBPB could be safely and successfully employed in the differential diagnosis of OP that affects the centrilobular area of a secondary lobule. Numerous publications have confirmed the utility of RP EBUS not only in localizing [13], but also in analyzing the internal structure [14] of peripheral pulmonary lesions. This has been shown not only in solid lesions, but more recently also in GGOs (pure and part-solid type), although with a lower diagnostic yield. Izumo et al. described RP EBUS images of GGOs [8]: the blizzard sign, as an enlarged and diffuse hyperintense acoustic shadow, for pure GGOs, and the mixed blizzard sign, as a diffuse heterogeneity with several hyperechoic dots, linear arcs and vessels, for part-solid GGOs. Other authors emphasized that the diagnostic yield increases if the probe is positioned within the lesion, rather than adjacent to it [13].

As for the sampling methods, transbronchial procedures have usually been performed under fluoroscopic guidance [15], with or without RP EBUS [3], and with or without guide-sheath (GS) $[13,16]$. Avoiding fluoroscopy, however, avoids unnecessary exposure to radiation, while the absence of GS allows reaching the lesion more easily. Performing RP EBUS TBPB without fluoroscopy [17] and without GS [16] is therefore preferable. In the cases presented in this study we have diagnosed OP with RP EBUS TBPB, without a radiological guide but just computing the distance to the target lesion, with a videobronchoscope provided with a wide working channel $(3.2 \mathrm{~mm})$ suitable for a quite large biopsy forceps, thus sampling enough tissue to perform a histological evaluation.

We acknowledge that both RP EBUS and lung biopsy (cryobiopsy/surgical) have each some pros and cons. RP EBUS is associated with fewer risks and/or complications than surgical biopsy, and RP EBUS TBPB could be considered as a first step in the diagnostic workup for some kinds of DPLDs such as OP [18]. On the other hand, lung biopsy and the transthoracic approach have a higher diagnostic yield. Indeed, a careful analysis of the radiological presentation is extremely important in selecting the best-suited procedure, since pathologies characterized by peribronchiolar and alveolar filling patterns are more easily visualized and more properly sampled by RP EBUS TBPB than those with a fibrotic pattern. Nonetheless, we believe that the specimens obtained by cryoprobes could improve the diagnostic yield also in this setting [19]. Lastly, the presence of fluoroscopic guidance and GS do not seem to be key in the diagnostic workup of pulmonary nodules, probably because pluri-segmental diseases offer the opportunity of exploring more bronchiolar routes. In our opinion, RB EBUS TBLB is neither a "stairway to heaven" [20], nor a "road to nowhere" [21], but simply a step on a "long and winding road" [22].

\section{References}

1. British Thoracic Society. The diagnosis, assessment and treatment of diffuse parenchimal lung diseases in adults. Thorax 1999;54:S1-28.

2. American Thoracic Society/European Respiratory Society. 
International Multidisciplinary Consensus Classification of the Idiopathic Interstitial Pneumonias. Am J Respir Crit Care Med 2002; 165:277-304.

3. Herth FJ, Ernst A, Becker HD. Endobronchial ultrasoundguided transbronchial lung biopsy in solitary pulmonary nodules and peripheral lesions. Eur Respir J 2002;20:972-4.

4. Kim EJ, Kim KC. Utility of radial probe endobronchial ultrasound-guided transbronchial lung biopsy in diffuse lung lesions. Tuberc Respir Dis 2019;82:201-10.

5. Lazor R. Organizing pneumonias. In: Cottin V, Cordier JF, Richeldi L, editors. Orphan lung diseases. A Clinical guide to rare lung disease. London: Springer; 2015; p. 363-378.

6. Raj R, Raparia K, Lynch DA, Brown KK. Surgical lung biopsy for interstitial lung diseases. Chest 2017;151:1131-40.

7. Hutchinson JP, Fogarty AW, McKeever TM, Hubbard RB. In hospital mortality after surgical lung biopsy for interstitial lung disease in the United States. 2000 to 2011. Am J Respir Crit Care Med 2016;193:1161-7.

8. Izumo T, Sasada S, Chavez C, Matsumoto Y, Tsuchida T. Radial endobronchial ultrasound images for ground-glass opacity pulmonary lesions. Eur Respir J 2015;45:1661-8.

9. Sasada S, Izumo T, Chavez C, Tsuchida T. Blizzard sign as a specific endobronchial ultrasound image for ground glass opacity: A case report. Resp Med Case Rep 2014;12:19-21.

10. Ali MS, Trick W, Mba BI, Mohananey D, Sethi J, Musani AI. Radial endobronchial ultrasound for the diagnosis of peripheral pulmonary lesions: a systematic review and metaanalysis. Respirology 2017;22:443-53.

11. Poletti V, Chilosi M, Olivieri D. Diagnostic invasive procedures in diffuse infiltrative lung diseases. Respiration 2004;71:107-19.

12. Gupta A, Youness H, Dhillon SS, Harris K. The value of using radial endobronchial ultrasound to guide transbronchial lung cryobiopsy. J Thorac Dis 2019;11:329-34.

13. Kurimoto N, Miyazawa T, Okimasa S, Maeda A, Oiwa H, Miyazu Y, et al. Endobronchial ultrasonography using a guide sheath increases the ability to diagnose peripheral pulmonary lesions endoscopically. Chest 2004;126:959-65.

14. Kurimoto N, Murayama M, Yoshioka S, Nishisaka T. Analysis of the internal structure of peripheral pulmonary lesions using endobronchial ultrasonography. Chest 2002;122:1887-94.

15. Gasparini S, Ferretti M, Secchi EB, Baldelli S, Zuccatosta L, Gusella P. Integration of transbronchial and percutaneous approach in the diagnosis of peripheral pulmonary nodules or masses: experience with 1,027 consecutive cases. Chest 1995;108:131-7.

16. Chung YH, Lie CH, Chao TY, Wang YH, Lin AS, Wang JL, et al. Endobronchial ultrasonography with distance for peripheral pulmonary lesions. Respir Med 2007;101:738-45.

17. Yoshikawa M, Sukoh N, Yamazaki K, Kanazawa K, Fukumoto S, Harada M, et al. Diagnostic value of endobronchial ultrasonography with a guide sheath for peripheral pulmonary lesions without X-ray fluoroscopy. Chest 2007;131:1788-93.

18. Ma M, Li Y, Cai H-R, Ding J-J, Wang Y-S, Gao Y-J, et al. Radial probe endobronchial ultrasound-guided lung biopsy for histopathological diagnosis of cryptogenic organizing pneumonia. Chin Med J (Engl) 2017;130:236-8.

19. Gnass M, Filarecka A, Pankowski J, Soja J, Bugalho A, Szlubowski A. Transbronchial lung cryobiopsy guided by endobronchial ultrasound radial miniprobe in interstitial lung diseases: preliminary results of a prospective study. Pol Arch Intern Med 2018;128:259-62.

20. Led Zeppelin. Stairway to heaven. Led Zeppelin IV; 1969.

21. Talking Heads. Road to nowhere. Little Creatures; 1985.

22. The Beatles. The long and winding road. Let it Be; 1970

Received for publication: 24 March 2020. Accepted for publication: 4 June 2020.

This work is licensed under a Creative Commons Attribution-NonCommercial 4.0 International License (CC BY-NC 4.0).

CCopyright: the Author(s), 2020

Licensee PAGEPress, Italy

Multidisciplinary Respiratory Medicine 2020; 15:658

doi:10.4081/mrm.2020.658 